\title{
A state-of-the-art analysis of the dwarf irregular galaxy NGC 6822 $\star, \star \star$
}

\author{
F. Fusco ${ }^{1,4}$, R. Buonanno ${ }^{1,2}$, S. L. Hidalgo ${ }^{3,4}$, A. Aparicio ${ }^{3,4}$, A. Pietrinferni ${ }^{2}$, G. Bono ${ }^{1,5}$, \\ M. Monelli ${ }^{3,4}$, and S. Cassisi ${ }^{2}$
}

1 Università di Roma Tor Vergata, Via della Ricerca Scientifica 1, 00133 Rome, Italy e-mail: federica.fusco@roma2.infn.it

2 INAF - Osservatorio Astronomico di Teramo, Via Mentore Maggini, 64100 Teramo, Italy

3 Instituto de Astrofísica de Canarias, Calle Via Lactea, 38205 La Laguna, Tenerife, Spain

${ }^{4}$ Departamento de Astrofísica, Universidad de La Laguna, 38205 Tenerife, Spain

5 INAF - Osservatorio Astronomico di Roma, Via Frascati 33, 00040 Monte Porzio Catone, Italy

Received 18 November 2013 / Accepted 25 August 2014

\begin{abstract}
We present a detailed photometric study of the dwarf irregular galaxy NGC 6822 aimed at investigating the properties of its stellar populations and, in particular, the presence of stellar radial gradients. Our goal is to analyse the stellar populations in six fields, which cover the whole bar of this dwarf galaxy. We derived the quantitative star formation history (SFH) of the six fields using the IAC method, involving IAC-pop/MinnIAC codes. The solutions we derived show an enhanced star formation rate (SFR) in Fields 1 and 3 during the past $500 \mathrm{Myr}$. The SFRs of the other fields are almost extinguished at very recent epochs and. We study the radial gradients of the SFR and consider the total mass converted into stars in two time intervals (between 0 and $0.5 \mathrm{Gyr}$ ago and between 0.5 and 13.5 Gyr ago). We find that the scale lengths of the young and intermediate-to-old populations are perfectly compatible, with the exception of the young populations in Fields 1 and 3. The recent SF in these two fields is greater than in the other ones. This might be an indication that in these two fields we are sampling incipient spiral arms. Further evidence and new observations are required to prove this hypothesis. In addition, we derived the age-metallicity relations. As expected, the metallicity increases with time for all of the fields. We do not observe any radial gradient in the metallicity.
\end{abstract}

Key words. galaxies: individual: NGC 6822 - Local Group - galaxies: dwarf - galaxies: star formation

\section{Introduction}

NGC 6822 is a barred dwarf irregular galaxy (dIrr) of type Ir IV-V (van den Bergh 1998) belonging to the Local Group (LG). This dwarf galaxy has been the subject of many studies, since it is one of the nearest dIrrs to the Milky Way (MW). The metallicity of NGC 6822 has been estimated by several authors (e.g. Gallart et al. 1996a; Venn et al. 2001; Sibbons et al. 2012), with values ranging from $[\mathrm{Fe} / \mathrm{H}]=-1.92 \pm 0.35$ (Clementini et al. 2003) to $[\mathrm{Fe} / \mathrm{H}]=-0.49 \pm 0.22$ (Venn et al. 2001). Kirby et al. (2013) carried out a spectroscopic study on single stars in seven dwarf irregular galaxies of the LG, for which they provide new and highly accurate metallicity estimates. The mean value obtained for NGC 6822 is $[\mathrm{Fe} / \mathrm{H}]=-1.05$ with a dispersion of 0.49 dex.

NGC 6822 is affected by a moderate foreground extinction because of the low galactic latitude $\left(l=25.4^{\circ}, b=\right.$ $-18.4^{\circ}$, Mateo 1998). According to Schlegel maps of the Galaxy (Schlegel et al. 1998; updated in Schlafly \& Finkbeiner 2011), the reddening along the line of sight towards NGC 6822 is $E(B-V)=0.21$. Moreover, several authors have found a difference in reddening between the innermost and outermost

* Based on observations collected with the ACS on board the NASA/ESA HST.

$\star \star$ The photometric catalogue is only available at the CDS via anonymous ftp to cdsarc.u-strasbg. fr (130.79.128.5) or via http://cdsarc.u-strasbg.fr/viz-bin/qcat?]/A+A/572/A26 regions of NGC 6822 (Massey et al. 1995; Gallart et al. 1996a; Gieren et al. 2006). The estimates of the distance cover a fairly wide interval ranging from $(m-M)_{0}=23.31 \pm 0.02$ mag (Feast et al. 2012) to $(m-M)_{0}=23.71 \pm 0.14 \mathrm{mag}$ (Salaris \& Cassisi 1998). In a recent study, Fusco et al. (2012, hereinafter Paper I), based on the Hubble Space Telescope (HST) observations of Cannon et al. (2012, hereinafter C12), estimated the reddening for the central region and external regions respectively as $E(B-V)_{C}=0.37 \pm 0.02$ and $E(B-V)_{E}=0.30 \pm 0.03$ and derived a distance modulus $(m-M)_{0}=23.54 \pm 0.05$ mag, corresponding to $510 \pm 10 \mathrm{kpc}$.

The star formation history (SFH) of this galaxy was first studied by Gallart et al. (1996b,c), who focused on the spatial variation of the stellar population in NGC 6822. Their analysis indicates that in the past 100-200 Myr an enhancement of the SF activity occurred over the whole main body of this galaxy. The strength of this enhancement has been different from one region to the other. This was supported by $\mathrm{C} 12$, who studied the SFH of NGC 6822, based on deep HST data. They find that this galaxy formed more than $50 \%$ of the stars in the last $5 \mathrm{Gyr}$. The SFH has been consistent throughout NGC 6822 in the past 500 Myr, with an increase in the star formation rate (SFR) in the last $50 \mathrm{Myr}$.

NGC 6822 is well known for its complex morphology. de Blok \& Walter (2000) mapped the entire extended distribution of $\mathrm{H}$ I of NGC 6822, identifying a number of peculiar features. In particular they observe an $\mathrm{H}$ i cloud in the north-eastern region of this galaxy, and they suggest that this feature could be 
Table 1. Images in the three fields, with filter, exposure time (s), $\alpha$ (h), $\delta$ (deg).

\begin{tabular}{ccccc}
\hline \hline Field & Filter & Exp. time $(\mathrm{s})$ & $\alpha(\mathrm{h})$ & $\delta(\mathrm{deg})$ \\
\hline Field 1 & $F 475 W$ & 423.0 & 19.726 & -14.561 \\
Field 1 & $F 475 W$ & 463.0 & 19.726 & -14.561 \\
Field 1 & $F 814 W$ & 883.0 & 19.726 & -14.561 \\
Field 1 & $F 814 W$ & 463.0 & 19.726 & -14.561 \\
Field 2 & $F 475 W$ & 559.5 & 19.732 & -14.619 \\
Field 2 & $F 475 W$ & 559.5 & 19.732 & -14.619 \\
Field 2 & $F 814 W$ & 559.0 & 19.732 & -14.619 \\
Field 2 & $F 814 W$ & 559.0 & 19.732 & -14.619 \\
Field 3 & $F 475 W$ & 559.5 & 19.738 & -14.678 \\
Field 3 & $F 475 W$ & 559.5 & 19.738 & -14.678 \\
Field 3 & $F 814 W$ & 559.0 & 19.738 & -14.678 \\
Field 3 & $F 814 W$ & 559.0 & 19.738 & -14.678 \\
\hline
\end{tabular}

associated with an interacting companion system. In addition, de Blok \& Walter (2003) noted that the number of blue stars across the $\mathrm{H}$ i cloud was particularly large.

$\mathrm{C} 12$ further analysed the nature of this $\mathrm{H}$ I cloud. In their study the authors ruled out that the suspected putative companion, coincident with their Field 1, is a bona fide galaxy. This conclusion is based on the absence of an overdensity of red giant branch (RGB) stars in that region. However, the authors state that "an interaction with a gas cloud without an ancient stellar component remains a viable solution".

The aim of the present work is to extend the analysis of Paper I in three more fields along the bar of NGC 6822, and thus end up with the total number of six fields. The photometric dataset is the same as for $\mathrm{C} 12$, but we perform a completely independent analysis, both in tools and method. In this paper the names of the fields coincide with those of C12. In particular, we investigate whether the nature of the suggested putative companion of NGC 6822 corresponding to Field 1 is confirmed. To our goal, we derive the SFH and age-metallicity relations (AMR) for the six fields, using the most updated version of the BaSTI evolutionary library ${ }^{1}$ (Pietrinferni et al. 2004) and IAC-pop/MinnIAC population synthesis code (Aparicio \& Hidalgo 2009; Hidalgo et al. 2011).

The paper is organized as follows. In Sect. 2 the observations and dataset are described; in Sect. 3 we analyse the stellar populations identified in this galaxy; in Sect. 4 we describe the SFH code, the input parameters, and the main results. The conclusions are presented in Sect. 5.

\section{Observations and data reduction}

The present photometry is based on archival HST datasets collected with the Advanced Camera for Surveys (HST GO programme 12180, P.I. Cannon). The same nomenclature as C12 (Fig. 1) is adopted here. In particular, we present here the analysis of three fields located in the north-western regions which are labelled Fields 1, 2, 3 from the outermost to the innermost. Field, filter, exposure time and coordinates of our dataset are listed in Table 1.

The images were prereduced using the standard HST pipeline. We performed photometry on individual images using DAOPHOT (Stetson 1987) and then simultaneous photometry on the four images of each field using ALLFRAME (Stetson 1994). At the end we obtained three catalogues - one

\footnotetext{
1 The full library is available at http://basti.oa-teramo.inaf. it
}

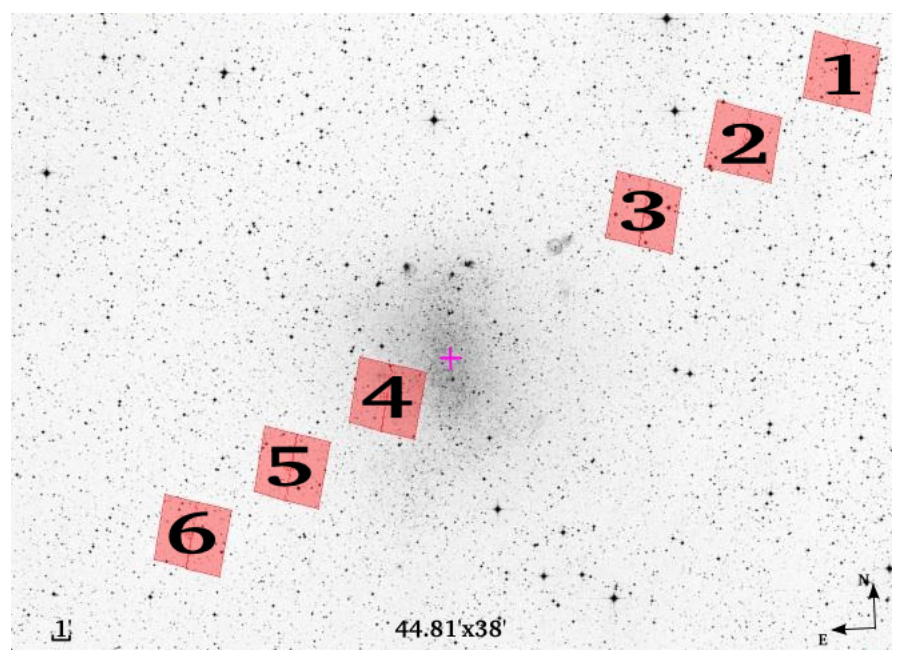

Fig. 1. Position of the six fields with the associated label considered in the present paper. The cross indicates the position of the centre of NGC 6822, whereas the scale, the dimension of the field, and orientation are indicated. The position of Field 1 coincides with the $\mathrm{H}$ I cloud identified by de Blok \& Walter (2000).

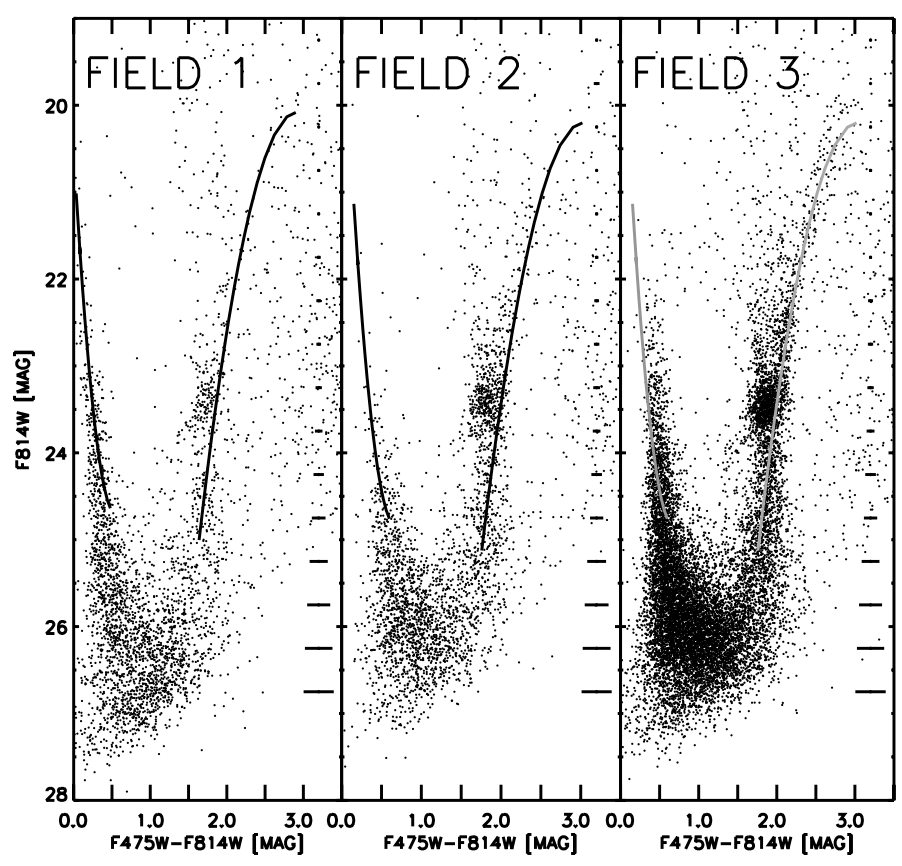

Fig. 2. Final $F 814 W, F 475 W-F 814 W$ CMDs, for Fields 1 , 2, and 3. The associated photometric errors are also shown. Superimposed on the CMDs are the ridge lines of the CMD of Field 4 determined in Paper I.

for each field, containing $\sim 15000, \sim 3500$, and $\sim 4000$ stars, respectively, from the innermost to the outermost field. The data were transformed to the standard VEGAMAG photometric system (Sirianni et al. 2005) using the most updated zeropoints ${ }^{2}$.

We thus obtained three $F 814 W, F 475 W-F 814 W$ CMDs, for Fields 1, 2, and 3. These CMDs are shown in Fig. 2. The CMD of Field 4 (for a detailed analysis see Paper I) is given in the same figure. We refer to Paper I for the CMDs of Fields 5 and 6.

The reddenings of Fields 4, 5, and 6 are given in Paper I. To estimate the reddenings of the Fields 1, 2, and 3 we superimposed on the CMDs the ridge lines of Field 4 already

\footnotetext{
2 Available at http://www.stsci.edu/hst/acs/analysis/ zeropoints
} 


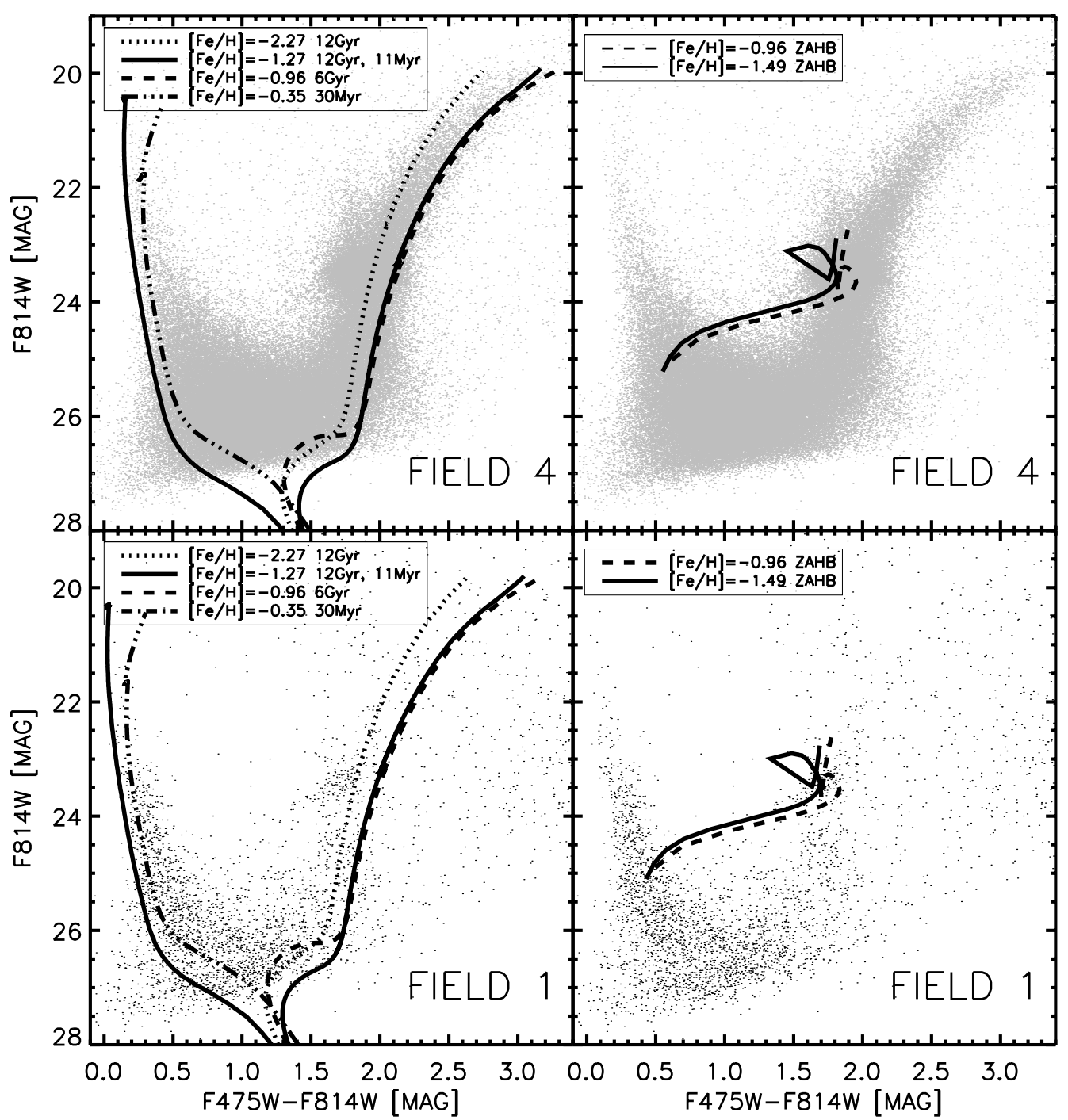

Fig. 3. Left panel: comparison between the observed CMDs for Fields 4 (upper panel) and 1 (lower panel) and scaled solar theoretical isochrones for different assumptions about age and metallicity. Lines on the RGB are for $[\mathrm{Fe} / \mathrm{H}]=-1.27,12 \mathrm{Gyr}$ (continuous line), $[\mathrm{Fe} / \mathrm{H}]=-0.96,6 \mathrm{Gyr}$ (dashed line), and $[\mathrm{Fe} / \mathrm{H}]=-2.27,12 \mathrm{Gyr}$ (dotted line). Lines on the MS show two isochrones, respectively with $[\mathrm{Fe} / \mathrm{H}]=-1.27$ and age $11 \mathrm{Myr}$ (solid line) and with $[\mathrm{Fe} / \mathrm{H}]=-0.35$ and age $30 \mathrm{Myr}$ (dashed-dot-dot-dot line). Right panel: comparison between core He-burning stars in the CMDs of Fields 1 and 4 and a theoretical ZAHB (with mass range between $0.6 M_{\odot}$ and $2.8 M_{\odot}$, in order to include both low-mass HB models and $\mathrm{RC}$ ones) for two different metallicities, $[\mathrm{Fe} / \mathrm{H}]=-0.96$, and -1.49 (dashed and continuous line respectively). All the shown isochrones have been retrieved from the BaSTI archive except the one corresponding to $[\mathrm{Fe} / \mathrm{H}]=-1.27$ and age of $11 \mathrm{Myr}$, which has been computed for the present work.

determined in Paper I (solid lines in Fig. 2). Thus, the absolute reddening in Field 1 turns out to be the same as in Fields 5 and 6, whereas Fields 2 and 3 have the same absolute reddening as Field 4 . The last two lines of Table 2 show the distance modulus $(m-M)_{F 814 W}$ and the $E(F 475 W-F 814 W)$ reddening value for the six fields. We assumed the same reddening error of Fields 5 and 6 for Fields 1, 2, and 3.

\section{Preliminary findings on the stellar populations in NGC 6822}

To perform a preliminary analysis of the possible presence of different stellar populations across NGC 6822 we superimposed a set of isochrones to the CMDs of Fields 1 and 4 (i.e. the outermost and innermost fields). We adopted scaled solar isochrones from the BaSTI library (Pietrinferni et al. 2004), and we used the distance modulus and reddening estimates discussed in Sect. 2. The result is shown in Fig. 3.

The comparison with the isochrones allows us to obtain some preliminary estimate of the age and metallicity of the stellar populations in NGC 6822:

- The two isochrones for $[\mathrm{Fe} / \mathrm{H}]=-1.27$, age $11 \mathrm{Myr}$, and $[\mathrm{Fe} / \mathrm{H}]=-0.35$, age $30 \mathrm{Myr}$, shown in Fig. 3, appear to constrain the blue edge of the main sequence (MS) fairly well. This comparison strongly suggests that NGC 6822 was still forming stars, at least in these two fields, in a very recent epoch.

- The slope and the location of the RGB in the CMD, on the other hand, give a strong constraint on the metallicity. The RGB of NGC 6822 shows a significant spread, larger than the expected colour error at the magnitude of this branch, as shown in Fig. 2. We conclude that a spread in metallicity or a combined spread in age and metallicity is present in 
this dwarf galaxy. The slope of the RGB mainly depends on the metallicity, but we cannot exclude an age effect. Younger RGB stars attain bluer colours, thus mimicking a more metal-poor stellar population. From Fig. 3 (left panels) it appears that the blue edge of the RGB is represented well by the $[\mathrm{Fe} / \mathrm{H}]=-2.27$ and $12 \mathrm{Gyr}$ isochrone. On the other hand, the red edge is compatible with intermediate-age isochrones with slightly higher metallicity (i.e. $[\mathrm{Fe} / \mathrm{H}]=-1.27$ and $[\mathrm{Fe} / \mathrm{H}]=-0.96)$. Therefore the age-metallicity degeneracy on the RGB does not allow us to constrain the spread in age and metallicity of the population in NGC 6822. The qualitative comparison between theoretical isochrones and observational data discloses that the photometric limit does not allow us to detect the MS turn off (TO) of the oldest population.

- In the righthand panels of Fig. 3 the zero age horizontal branch (ZAHB) is shown for two metallicities $[\mathrm{Fe} / \mathrm{H}]=-0.96$ and $[\mathrm{Fe} / \mathrm{H}]=-1.49$ (mass range between $0.6 M_{\odot}$ and $2.8 M_{\odot}$ ). The observed red clump appears well fitted by the central helium-burning sequence of $[\mathrm{Fe} / \mathrm{H}]=-0.96$. There is some evidence that the intermediate-red colour HB is partially populated. The presence of the bright blue MS does not allow us to detect any blue HB stars.

To have a closer insight into the nature of the stellar populations in the different fields of NGC 6822 we performed star counts in the different branches of the CMDs, including the three fields of Paper I. We started by selecting five boxes in the most populated regions in the CMD of Field 4. These were defined as

UpMS: the upper MS box encloses MS stars brighter than $F 814 W=23.7$ mag where the MS luminosity function (LF) shows a slight change in its slope.

LowMS: the lower main sequence box contains MS stars between $25 \mathrm{mag}<F 814 \mathrm{~W}<23.7 \mathrm{mag}$. According to the completeness tests we performed, $F 814 W=25 \mathrm{mag}$ is the limit of our photometry. The cut at $F 814 \mathrm{~W}=$ 23.7 mag corresponds to the TO of the stars with an age of $450 \mathrm{Myr}$ (for $[\mathrm{Fe} / \mathrm{H}]=-0.66$ ). The comparison between UpMS and LowMS allows us to discriminate between stars younger and older than $450 \mathrm{Myr}$.

RC: according to the change in slope of the RGB LF, we defined the interval $23 \mathrm{mag} \leq F 814 \mathrm{~W} \leq 23.9 \mathrm{mag}$ as the red clump region.

LowRGB: the lower RGB corresponds to 23.9 mag $\leq F 814 \mathrm{~W} \leq$ $25 \mathrm{mag}$. The brighter limit in magnitude is set as the boundary of the RC region, while the fainter limit coincides with the limit in magnitude selected for our photometry according to the completeness tests.

UpRGB: correspondingly we define the upper RGB branch as the region of the RGB brighter than the RC. The bluer part of the RGB was excluded with a by-eye inspection in order to minimize the contribution of the asymptotic giant branch to the star counts.

Table 2 shows the star counts in each box and the relative ratios. The errors listed in this table are given by the statistical fluctuations of the counts. We performed several experiments of artificial stars in all the fields (for a complete description see Sect. 4.1) in order to confirm the statistical completeness of the star counts. The result is shown in Fig. 4, confirming that the counts at the limit $F 814 \mathrm{~W}=25 \mathrm{mag}$ are complete at least at the $85 \%$ level.

To perform quantitative comparisons, the box boundaries were shifted according to the relative reddening of each field.
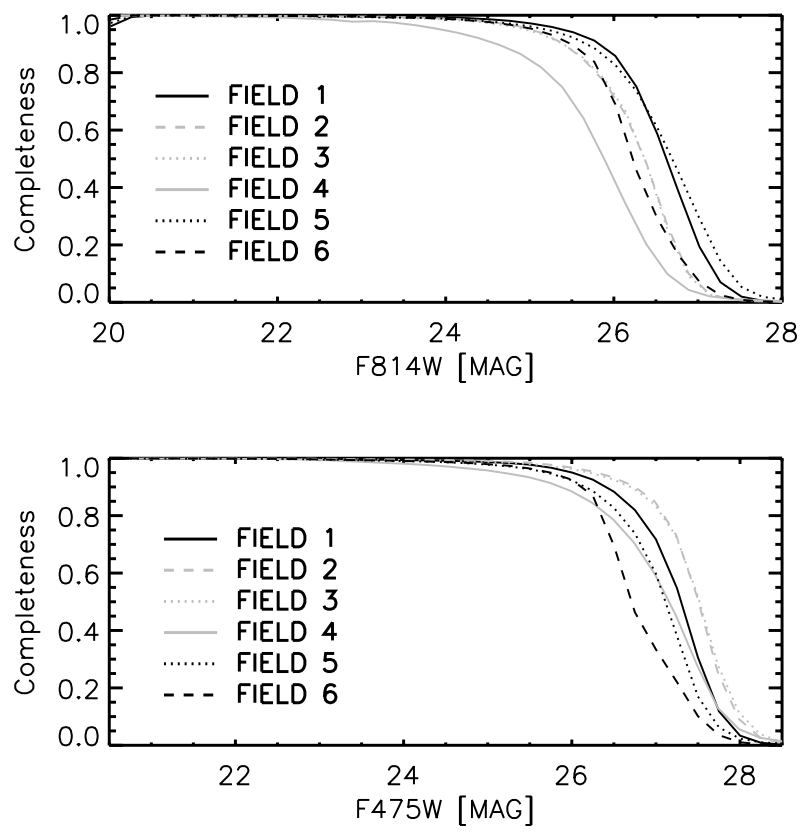

Fig. 4. Result of the artificial star tests in all the fields for the two filters, $F 814 W$ (upper panel) and $F 475 W$ (lower panel). See text for a full description.

Inspection of Table 2 immediately reveals that the two ratios UpMS/UpRGB and LowMS/LowRGB, i.e. the number of stars on the MS with respect to those on the RGB at the same luminosity level, turn out to be definitely higher in Field 1, and marginally in Field 3, than in all the other fields. The completeness of the star counts in the boxes of the first ratios is nearly $100 \%$, hence, highly significative. These ratios, taken at their face values, could provide a first hint that the stellar populations in Field 1 and 3 could be peculiar owing to the underabundace of RGB stars with respect to MS stars. In particular, this result could apparently suggest that a very recent burst of star formation took place in Field 1, which was more intense than in the other fields, including the innermost Field 4.

Therefore we computed the cumulative LFs of the RGB and of the MS for the six fields, as shown in Fig. 5. While the RGB LFs appear virtually identical in all of the fields, the LF of Field 1 starts increasing at brighter magnitudes for the MS with respect to all the other fields. This is a second hint to support the idea that the star formation in Field 1 could somehow be peculiar and that a relatively recent burst of star formation could have occurred in this region.

\section{A detailed analysis of the SFH in the different fields}

The former findings indicate that significant differences may exist in the stellar populations of the six fields. To investigate these differences in depth, we use resolved population synthesis tools to calculate the SFH of the six fields. To obtain the SFH solution we considered only the stars with $\sigma_{F 814 W} \leq 0.2, \sigma_{F 475 W} \leq 0.2$, and $\mid$ sharpness $\mid \leq 0.5^{3}$. This selection allows us to obtain the solution using only stellar objects and thus improving the precision of the SFHs.

\footnotetext{
The sharpness parameter is a standard DAOPHOT output, which quantifies the accuracy of the PSF profile.
} 
F. Fusco et al.: The SFH of dIrr galaxy NGC 6822

Table 2. Star counts and corresponding number ratios in the selected boxes of the CMD for the various fields of NGC 6822.

\begin{tabular}{ccccccc}
\hline \hline & Field 1 & Field 2 & Field 3 & Field 4 & Field 5 & Field 6 \\
\hline Region & $N_{1} \pm \epsilon_{N 1}$ & $N_{2} \pm \epsilon_{N 2}$ & $N_{3} \pm \epsilon_{N 3}$ & $N_{4} \pm \epsilon_{N 4}$ & $N_{5} \pm \epsilon_{N 5}$ & $N_{6} \pm \epsilon_{N 6}$ \\
\hline LowMS & $257 \pm 16$ & $160 \pm 12$ & $1146 \pm 33$ & $5047 \pm 71$ & $857 \pm 29$ & $477 \pm 21$ \\
UpMS & $86 \pm 9$ & $16 \pm 4$ & $188 \pm 13$ & $779 \pm 27$ & $79 \pm 8$ & $77 \pm 8$ \\
LowRGB & $106 \pm 10$ & $219 \pm 14$ & $708 \pm 26$ & $10191 \pm 100$ & $2435 \pm 49$ & $537 \pm 23$ \\
UpRGB & $62 \pm 7$ & $105 \pm 10$ & $403 \pm 20$ & $6223 \pm 78$ & $1313 \pm 36$ & $322 \pm 17$ \\
\hline Ratio & $R_{1} \pm \epsilon_{R 1}$ & $R_{2} \pm \epsilon_{R 2}$ & $R_{3} \pm \epsilon_{R 3}$ & $R_{4} \pm \epsilon_{R 4}$ & $R_{5} \pm \epsilon_{R 5}$ & $R_{6} \pm \epsilon_{R 6}$ \\
\hline UpMS/UpRGB & $1.4 \pm 0.2$ & $0.15 \pm 0.04$ & $0.47 \pm 0.03$ & $0.12 \pm 0.01$ & $0.06 \pm 0.01$ & $0.24 \pm 0.03$ \\
LowMS/LowRGB & $2.4 \pm 0.2$ & $0.73 \pm 0.06$ & $1.56 \pm 0.05$ & $0.49 \pm 0.01$ & $0.35 \pm 0.01$ & $0.89 \pm 0.04$ \\
LowMS/UpMS & $3.0 \pm 0.5$ & $10.0 \pm 0.8$ & $13.6 \pm 0.4$ & $6.5 \pm 0.1$ & $10.8 \pm 0.4$ & $6.2 \pm 0.3$ \\
\hline$(m-M)_{F 814 W}$ & $24.1 \pm 0.1$ & $24.2 \pm 0.1$ & $24.2 \pm 0.1$ & $24.2 \pm 0.1$ & $24.1 \pm 0.1$ & $24.1 \pm 0.1$ \\
$E(F 475 W-F 814 W)$ & $0.51 \pm 0.05$ & $0.63 \pm 0.05$ & $0.63 \pm 0.05$ & $0.63 \pm 0.04$ & $0.51 \pm 0.05$ & $0.51 \pm 0.05$ \\
\hline
\end{tabular}

Notes. The distance modulus and the absolute reddening of the fields are listed.

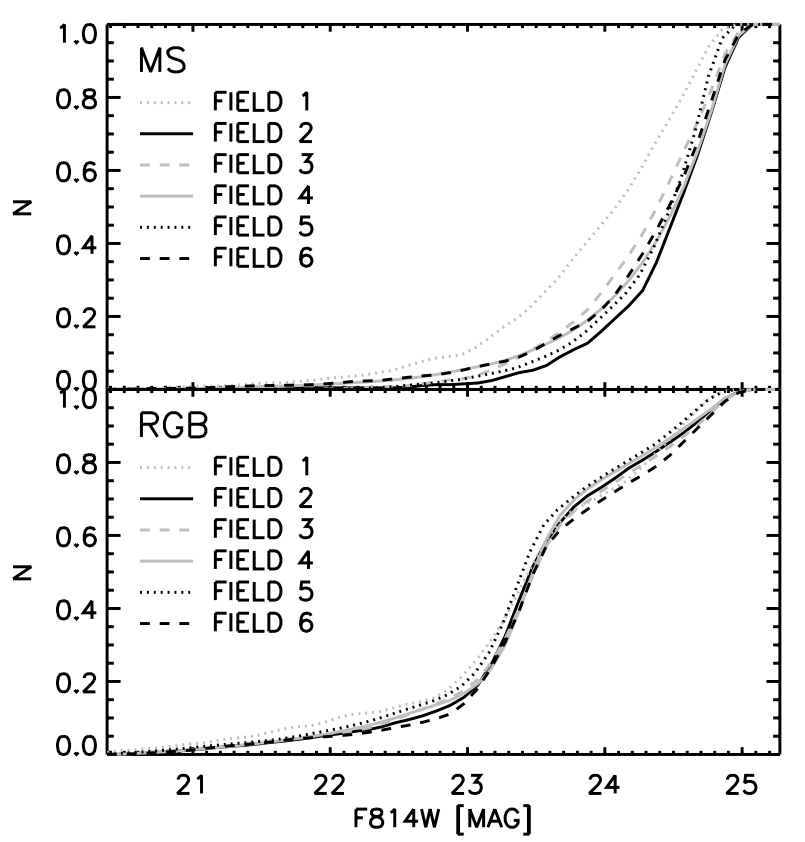

Fig. 5. Cumulative LF of the MS (Upper panel) and of the UpRGB (Lower panel) for the six fields considered. The LF of the RGB in the interval $22.7 \leq F 814 \mathrm{~W} \leq 23.7$, corresponding to the $\mathrm{RC}$ region, has been obtained by interpolation along the RGB LF at magnitudes slightly fainter and brighter than the $\mathrm{RC}$ box.

The first step is to accurately represent the observational effects which is done through tests of artificial stars (Stetson \& Harris 1988). We injected 10000000 stars in each image in 150 iterations. The stars were distributed uniformly in the images, and placed at a fixed relative distance set at $2 R_{\mathrm{PSF}}+1$ pixel, where $R_{\mathrm{PSF}}$ is the PSF radius in pixels. Once the stars had been injected in the images, we performed a new photometry, with the same procedure as was used to reduce the original images.

\subsection{Theoretical framework and input parameters}

The SFH was derived by applying the state-of-art package IACstar/IAC-pop/MinnIAC (Aparicio \& Gallart 2004; Aparicio \& Hidalgo 2009; Hidalgo et al. 2011), specifically developed for analysing resolved stellar populations. Even though the SFH of the various fields of NGC 6822 has been already studied by $\mathrm{C} 12$, the population synthesis code we are using for the present analysis is completely independent of the one adopted in the former analysis. At the same time, it is based on an independent, updated library of stellar models. Therefore, this investigation is also a test of the consistency of SFH obtained with independent population synthesis codes and stellar model libraries.

In short, IAC-star is used to create a synthetic CMD model. In our case, the synthetic CMD model contains $10^{7}$ stars with a constant SFR between 0 and $13.5 \mathrm{Gyr}$ and uniform metallicity distribution between $Z=0.0001$ and $Z=0.01$ - corresponding to $[\mathrm{Fe} / \mathrm{H}]=-2.27$ and $[\mathrm{Fe} / \mathrm{H}]=-0.27$. The extreme values were selected to cover all the ages and all the metallicities (Clementini et al. 2003; Venn et al. 2001) of the stars present in NGC 6822. We selected the BaSTI stellar evolution library (Pietrinferni et al. 2004), a Kroupa initial mass function (Kroupa 2001), and a 30\% binary fraction. After a suitable simulation of the observational effects in the synthetic CMD, the SFH is obtained using IAC-pop/MinnIAC algorithms (Aparicio \& Hidalgo 2009; Hidalgo et al. 2011) by comparison of the distribution of stars in the synthetic CMD with that in the observed CMD. The age bins selected are of 0.1 Gyr for age $<0.2 \mathrm{Gyr}, 0.3 \mathrm{Gyr}$ for $0.2<$ age $<0.5 \mathrm{Gyr}$, 0.25 Gyr for $0.5<$ age $<1 \mathrm{Gyr}, 1 \mathrm{Gyr}$ for $2<$ age $<5 \mathrm{Gyr}$, and $8.5 \mathrm{Gyr}$ for $5<$ age $<13.5 \mathrm{Gyr}$. The metallicity bins selected are $3 \times 10^{-4}$ for $10^{-4} \leq \mathrm{Z} \leq 10^{-3}, 2 \times 10^{-3}$ for $10^{-3} \leq \mathrm{Z} \leq 3 \times 10^{-3}, 4 \times 10^{-3}$ for $3 \times 10^{-3} \leq \mathrm{Z} \leq 7 \times 10^{-3}$, and $3 \times 10^{-3}$ for $7 \times 10^{-3} \leq \mathrm{Z} \leq 10^{-2}$. These values were selected according to the age-metallicity resolution tests presented in Sect. 4.2. With this method we obtain the smoothed SFHs, which include 24 single SFHs. For a full description we refer to Hidalgo et al. (2011).

To minimize the effect of the estimates in the external parameters, namely distance, reddening, and photometric zeropoints, IAC-pop/MinnIAC synthesis codes provide an offset both in magnitude and colour. These values are those which minimize the $\chi^{2}$ of the solution. Table 3 shows the distance of the fields from the centre of NGC 6822, the $\chi^{2}$, and the offset in colour and magnitude of the solutions.

To evaluate the relative importance of the SFR in the fields, we evaluated the mass fraction of stars produced in two age bins. The results are listed in Table 3, where the old-intermediate- (between 13.5 and $0.5 \mathrm{Gyr}$ ago) and young- (from $0.5 \mathrm{Gyr}$ ago to date) epochs were taken into account. Inspection of this table reveals that the integral $\int_{0.5}^{13.5} \psi(t) \mathrm{d} t$ in all the fields is comparable, whereas the integral $\int_{0}^{0.5} \psi(t) \mathrm{d} t$ turns out to be very small for all the fields with the exception of Fields 1 and 3. In these 


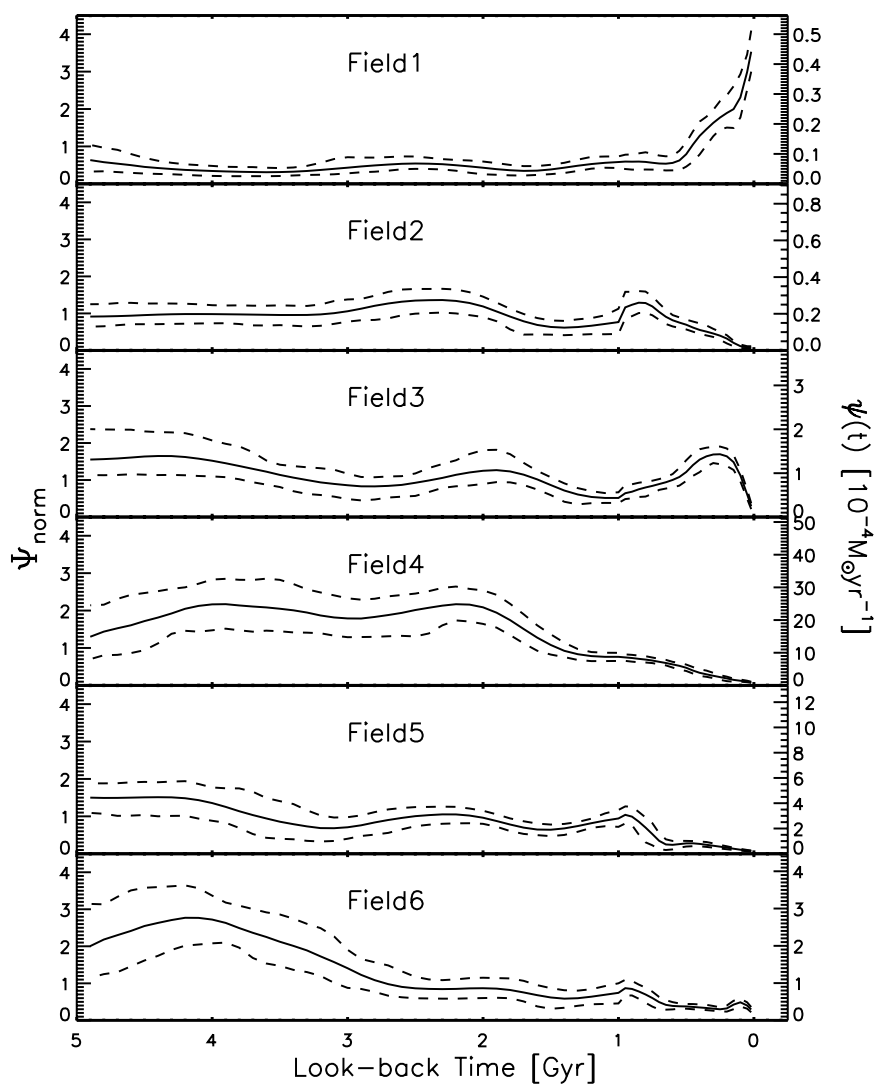

Fig. 6. SFH for the six fields (solid line) as function of the time for the last $5 \mathrm{Gyr}$, with the relative error (dashed line). $\psi(t)$ (labelled of the right-side axis) is the stellar mass formed, while $\Psi_{\text {norm }}$ (labelled of the left-side axis) shows $\psi(t)$ normalized to $\int_{0}^{13.5} \psi(t) \mathrm{d} t$. See text.

fields the SFR shows a slight enhancement in this time interval. Nine and six times more stars were produced in Fields 1 and 3, respectively, than in Field 4.

In addition, the mean metallicity of the six fields is listed in Table 3 . This result is perfectly compatible with the metallicity estimates by Kirby et al. (2013). Moreover, the associated spread in metallicity is compatible with the spectroscopic results.

\subsection{SFH solution and $A M R$}

The SFH solution of the fields is shown in Fig. 6. The function $\psi(t)$ is the stellar mass formed as a function of time, while $\Psi_{\text {norm }}$ corresponds to $\psi(t)$ normalized to $\int_{0}^{13.5} \psi(t) \mathrm{d} t$. The scales along the vertical axis are different for each field as a consequence of the difference in the total stellar mass. The solutions we obtained are consistent with the results presented by $\mathrm{C} 12$. Figure 7 shows observed, solution and residual CMDs in the case of Field 5.

The photometric limit avoids properly sampling the MS TO of the oldest population. To estimate the age limit of our solution, we carried out age resolution tests (for a full description we refer to Hidalgo et al. 2011). Using IAC-star we created six mock populations of 10, 5, 3, 1, 0.2, $0.1 \mathrm{Gyr}$. Thus we performed the same procedure adopted to obtain the SFH solution, in order to recover the narrow mock bursts at different times. The result is shown in Fig. 8 for Field 2, as an example. Scrutiny of this figure shows that we are not able to recover the mock bursts of 5 and 10 Gyr. For this reason, the temporal scales of our solutions are not reliable for ages older than about 5 Gyr, although the integral $\int_{5}^{13.5} \psi(t) \mathrm{d} t$ is still accurate and well defined.
We now concentrate on the analysis of the individual SFH solutions (Fig. 6) taking only ages $\leq 5 \mathrm{Gyr}$ into account. This analysis discloses the following results:

Field 1. This field does not show any significant burst of SF until $500 \mathrm{Myr}$ ago, when the most recent burst began and continued to increase up to a very recent epoch.

Field 2. This field shows an SFR that remains quite constant over the whole time interval and tends to zero at the most recent epochs.

Field 3. The SFR in Field 3 appears to be as constant as that of Field 2. In the past $500 \mathrm{Myr}$ the SF, according to our solution, maintains a higher rate than in the other fields, but tends to disappear at very recent epochs.

Field 4. A unique extended SF episode took place in this region of NGC 6822 between 5 and 1.5 Gyr ago. In the last 1.5 Gyr the SFR vanished.

Field 5. The SF calculated for this field is analogous to the rate obtained in Field 2.

Field 6. The SF calculated for this field is analogous to the one obtained in Field 4.

From the analysis of the single solutions of the SF, we find that in Fields 2, 4, 5, and 6, the SFR has been slowly decreasing in the last $1.5 \mathrm{Gyr}$, and it is exhausting at very recent epochs. Fields 1 and 3 are apparent exceptions to this frame. For these field the solutions show an enhancement in the SFR in the last $500 \mathrm{Myr}$, and in the case of Field 1 it has still been active at very recent epochs.

In addition, the IAC-pop/MinnIAC method allows deriving the AMR as a part of the SFH. The AMRs for six fields of NGC 6822 are shown in Fig. 9 for the last 5 Gyr.

The AMRs disclose that the metallicity grows with time in all the six fields, ranging between $-1.0 \lesssim[\mathrm{Fe} / \mathrm{H}] \lesssim-0.5$. According to our solution no difference in the AMR of the six fields is identified. These values are compatible with the mean metallicity and the spread in metallicity recently found spectroscopically for NGC 6822 by Kirby et al. (2013).

\subsection{Radial gradients}

Our dataset samples the bar of NGC 6822 up to about five times its scale length. This offers a good opportunity to investigate the radial stellar populations distribution and the possible presence of a population gradients.

Several authors have studied the population gradients in the dwarf galaxies of the LG (e.g. Hidalgo et al. 2013 and references therein). In all the cases the trend we found is the same: the age of the youngest populations gets older for increasing radius, and progressively younger populations are only found in the innermost regions. The observed effects agree with models of simulated dwarf galaxies (Schroyen et al. 2013).

Regarding NGC 6822, the spatial distribution of the stellar populations have already been discussed by Gallart et al. (1996b,c). They studied the central regions of the galaxy and found an enhancement of the SFR in the past 100-200 Myr, which occurred over the whole main body of the galaxy. The strength of this enhancement is different between the different regions of NGC 6822. This results were confirmed by $\mathrm{C} 12$, who noticed that NGC 6822 formed more than $50 \%$ of the stars in the last 5 Gyr.

In this research we started evaluating $\int \psi(t) \mathrm{d} t$ in two time intervals, between 0 and $0.5 \mathrm{Gyr}$ ago and between 0.5 and $13.5 \mathrm{Gyr}$ ago, as a function of the galactocentric distance. The results are shown in Fig. 10. Clear exponential profiles are disclosed in all 
Table 3. Details of the SFH solution for the six fields.

\begin{tabular}{cccccccc}
\hline \hline Field & Dist. [deg] $]^{a}$ & $\chi^{2 b}$ & $\Delta F 814 W^{c}$ & $\Delta(F 475 W-F 814 W)^{d}$ & $\int_{0}^{0.5} \psi(t) \mathrm{d} t^{e}$ & $\int_{0.5}^{13.5} \psi(t) \mathrm{d} t^{f}$ & $\langle[\mathrm{Fe} / \mathrm{H}]\rangle^{g}$ \\
\hline 1 & 0.43 & 0.78 & 0.20 & 0.05 & $0.100 \pm 0.007$ & $0.90 \pm 0.07$ & $-0.9 \pm 0.3$ \\
2 & 0.32 & 0.76 & 0.20 & 0.15 & $0.017 \pm 0.002$ & $0.98 \pm 0.06$ & $-0.8 \pm 0.1$ \\
3 & 0.22 & 1.04 & 0.10 & 0.05 & $0.066 \pm 0.004$ & $0.93 \pm 0.08$ & $-0.9 \pm 0.2$ \\
4 & -0.06 & 2.35 & 0.08 & 0.15 & $0.011 \pm 0.001$ & $0.99 \pm 0.08$ & $-0.7 \pm 0.1$ \\
5 & -0.16 & 1.26 & 0.00 & 0.07 & $0.009 \pm 0.001$ & $0.99 \pm 0.07$ & $-1.0 \pm 0.2$ \\
6 & -0.26 & 1.23 & 0.05 & 0.02 & $0.018 \pm 0.002$ & $0.98 \pm 0.09$ & $-1.0 \pm 0.1$ \\
\hline
\end{tabular}

Notes. ${ }^{(a)}$ Distance from the centre of each field, where the negative values identify the fields in the south-eastern region of the galaxy. ${ }^{(b)} \chi^{2}$ of the SFH solution. ${ }^{(c)}$ Offsets in $F 814 W$ of the solution. ${ }^{(d)}$ Offsets in $(F 475 W-F 814 W)$ of the solution. ${ }^{(e)}$ Integral of the SFR between 0.5 and13.5 Gyr ago. ${ }^{(f)}$ Integral of the SFR from $0.5 \mathrm{Gyr}$ ago to date. ${ }^{(g)}$ Mean metallicity and spread in metallicity of the solution.

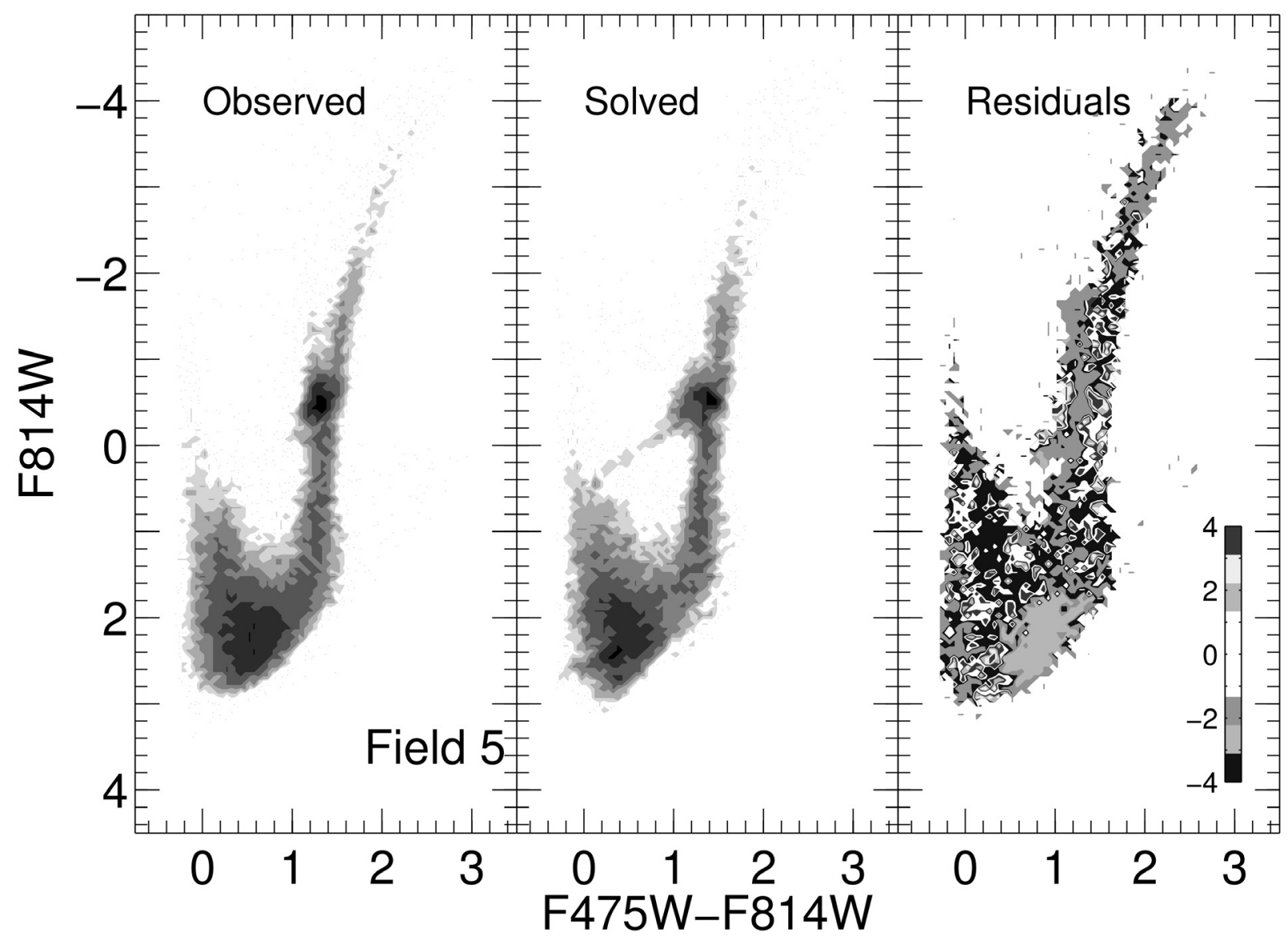

Fig. 7. Left panel: observed CMD for Field 5. Central panel: the CMD according to the SF produced by IAC-pop/MinnIAC. Right panel: residuals obtained as the difference between the observed and the solution CMDs counted in different bins in units of Poisson errors. Different colours correspond to different errors in agreement with the scale shown in the right panel. A similar result is obtained for all the other fields.

cases, except for the young populations of Fields 1 and 3. The scale lengths are compatible within the errors for the old and young populations. These result in $r_{0.5}^{13.5}=680 \pm 75 \mathrm{pc}$ and $r_{0}^{0.5}=730 \pm 160 \mathrm{pc}$ for the old- and the young-population. $r_{0}^{0.5}$ was computed excluding Fields 1 and 3. Fields 1 and 3 show young populations that exceed the ones of the surrounding fields by a factors of 10 and 3, respectively. This may be the simple result of a stochastic enhancement of the SFR in recent epochs. However, this could also be the signature of incipient spiral arms. With the present dataset this is only a speculative suggestion. New data are required to either support or rule out this possibility.
Following Hidalgo et al. (2009), we consider the times corresponding to the 75 and 95 percentiles of the SFR to further analyse the radial gradients. In other words, the times $t_{75}$ such that $\int_{0}^{t_{75}} \psi(t) \mathrm{d} t / \int_{0}^{T} \psi(t) \mathrm{d} t=0.75$ and $t_{95}$ such that $\int_{0}^{t_{95}} \psi(t) \mathrm{d} t / \int_{0}^{T} \psi(t) \mathrm{d} t=0.95$, where $T$ is the current age of the system. Figure 11 shows the look-back time and the metallicity as a function of the galactocentric distance for the two percentiles. Scrutiny of this figure reveals that the 75 percentile corresponds to similar look-back times in all the cases. The lookback times associated to the 95 percentile are similar for Fields 2, 4,5 , and 6 , but they correspond to a more recent epoch for 


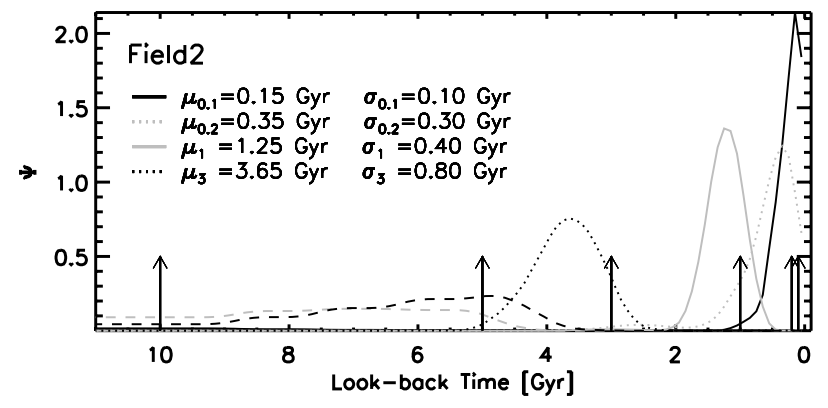

Fig. 8. Age resolution tests for Field 2 for six different burst ages (indicated by the arrows), namely 10, 5, 3, 0.2, 0.1 Gyr. Gaussian profiles represent the recovered SF episodes. The age peak and $\sigma$ are given. Each colour represents the recovered SF episode of a different age, in dashed-grey-, dashed-black-, dotted-black-, continuous-grey-, dottedgrey-, and continuous-black- lines respectively from the oldest to the youngest. Similar results are obtained for all the other fields.

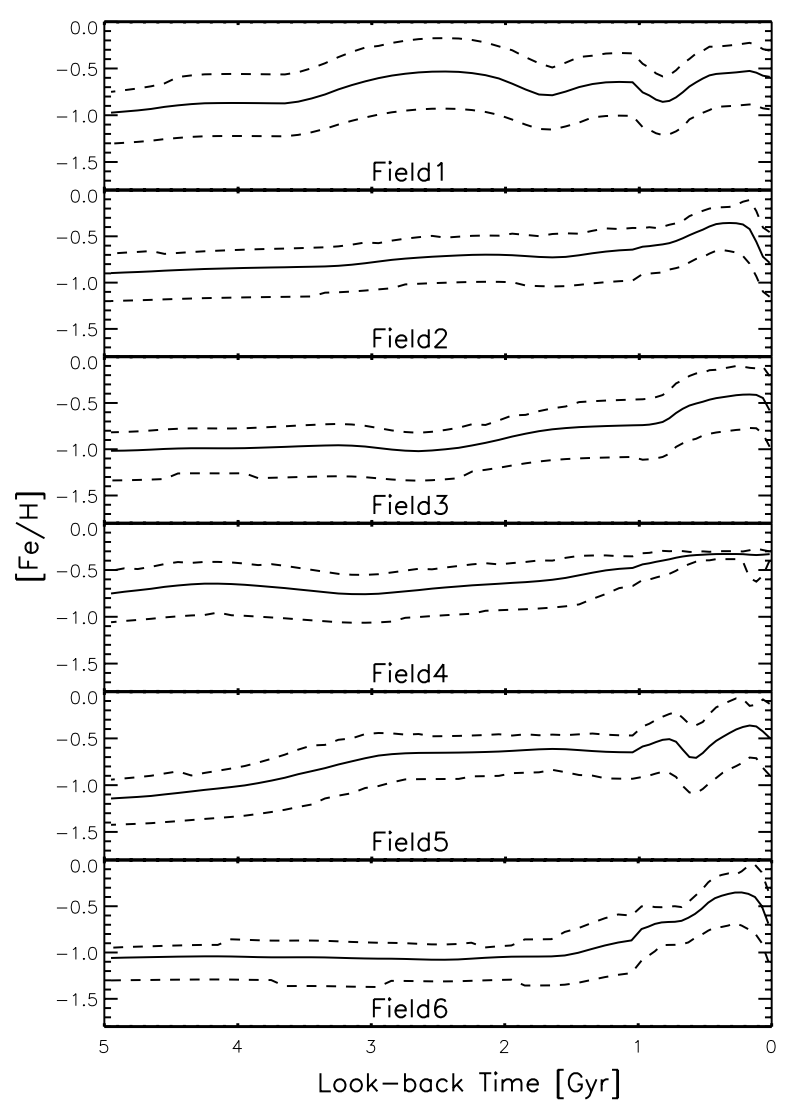

Fig. 9. AMRs for the six fields (solid lines), including error bars (dashed lines), as function of the time for the last 5 Gyr.

Fields 1 and 3. Moreover, Fig. 11 discloses that the metallicity increases in all the fields from the 75 to the 95 percentiles, but it does not show any radial gradient. To quantify these findings, we obtained an empirical relation $Z=Z(t, D)$, where $Z$ is the metallicity, $t$ is the look-back time, and $D$ the galactocentric distance in $\mathrm{kpc}$

$$
Z=[(5.7 \pm 1.5)-(0.57 \pm 0.08) t-(0.8 \pm 8.5) D] \times 10^{-3} .
$$

The errors of the fitting parameters confirm that the metallicity significantly increases with time, but that no significant metallicity gradient as a function of the distance is present. These results confirm the findings that have already been presented.

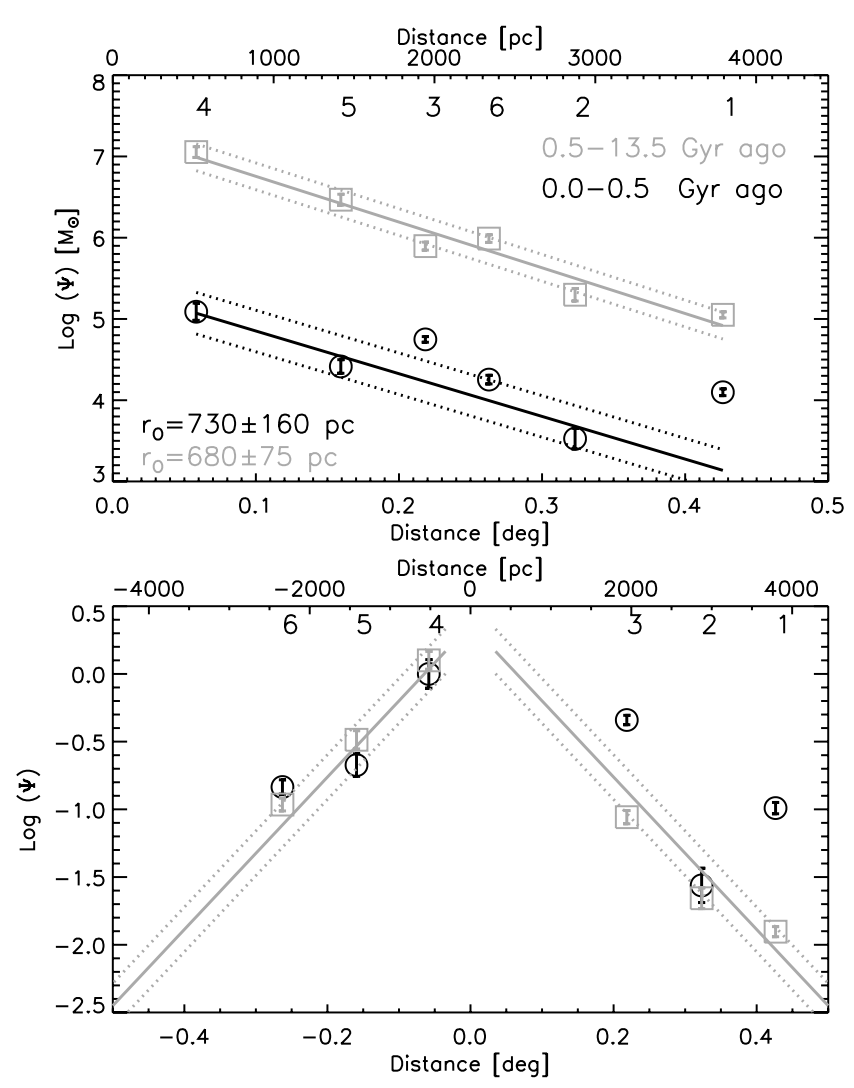

Fig. 10. Integral of the SFR, $\psi(t)$, for the young (from 0.5 Gyr to date; black dots) and intermediate-to-old (between 0.5 and 13.5 Gyr ago; grey squares) stellar populations, as a function of the galactocentric distance. Upper panel: the absolute value of the galactocentric distance is shown in the horizontal axis. Distances are given both in degrees (lower axis scale) and in parsecs (upper axis scale). Distances in parsecs have been obtained assuming a distance to NGC 6822 of $510 \mathrm{kpc}$ (Paper I). The solid lines show the best fit of the points in the two time intervals, with the associated errors (dotted lines). Fields 1 and 3 were excluded from the fit in the case of the young stellar population. The resulting scale length $r_{0}$ and the name of the field are indicated. Lower panel: same as the upper panel, but the galactocentric distances are shown as they appear along the galactic semi-major axis (negative and positive values corresponding to eastern and western fields, respectively). Points have been shifted vertically in order to make the older and younger distribution barycentres coincide. The vertical scale zeropoint is arbitrary. Grey lines reproduce the fit shown in the upper panel for the older population. The colour coding is the same a in the upper panel.

NGC 6822 does not show the trend found in similar galaxies by Hidalgo et al. (2013). They studied the SFH of two dIrr/dSph galaxies, namely LGS-3 and Phoenix, whose total stellar masses are about 100 times lower than that of NGC 6822. They find that, for ages $<9$ Gyr, the SFR gradually decreases outwards and that the metallicity does not change significantly with time or with distance from the centre. For recent epochs they find a steep increase in the metallicity, which does not have a counterpart in the SFR.

\section{Conclusions}

In this paper we have presented a new photometric analysis of three fields in the north-eastern region of the dIrr NGC 6822. We carried out a new and independent study of the SFH throughout this galaxy, considering both these three fields and the three fields presented in Paper I. The study was based on the IAC 


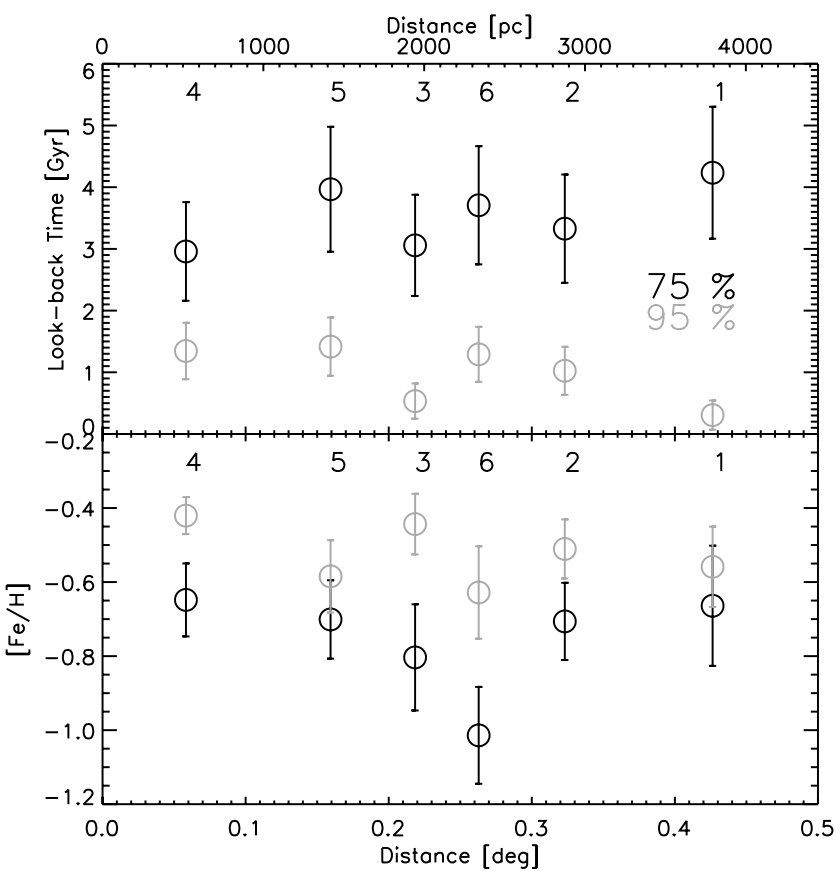

Fig. 11. Age and metallicity as a function of the galactocentric distance. The latter is shown both in degrees (lower axis scale) and in parsecs (upper axis scale). The distances in parsecs have been obtained assuming a distance to NGC 6822 of $510 \mathrm{kpc}$ (Paper I). Upper panel: look-back times of the 75 and 95 percentiles of the SFR as a function of the galactocentric distance. Numbers from 1 to 6 within the panel identify the corresponding fields. Lower panel: the mean metallicity for the same percentiles. The percentiles and the colour coding are the same as in the upper panel.

method, involving the IAC-pop/MinnIAC codes. As a consequence this work presents several new results for the SFRs that deepen some of the previous findings by $\mathrm{C} 12$. In addition, as an important new result, we provide the AMRs as a function of time.

The performed analysis provided the following results.

- The SFH obtained using this methodology reveals that Field 1 shows an enhancement in the SFR that began around $500 \mathrm{Myr}$ ago and has been increasing up to a very recent epoch. The SFR during this time interval is twice as high as the average for that field at earlier epochs. In all the other fields we find that the last Gyr was characterized by a slow decrease in the SFR. The AMRs show that the metallicity grows with time throughout the whole galaxy. The mean metallicity and the metallicity spread supports recent spectroscopic measurements by Kirby et al. (2013).

- We studied the radial gradients of the SFR. We considered the total mass converted into stars for young (between 0 and 0.5 Gyr ago) and intermediate-to-old (between 0.5 and 13.5 Gyr ago) ages. We observe a clear decreasing trend with the galactocentric distance in both cases, with very similar scale lengths. Fields 1 and 3 are an exception. The recent SF in these two fields clearly exceeds the one of the other fields. Nevertheless the intermediate-to-old populations follow the same trend as in the other fields. This can be considered as a sign that the intermediate-old stellar population is mixed well along the bar of NGC 6822, while this does not hold for young populations. Thus, young populations follows the same trend with the same scalelength as the intermediate-toold ones in all but two fields (Fields 1 and 3). This may indicate that in these two fields we are sampling incipient spiral arms. Nevertheless, this conclusion has to be considered as only hypothesis as long as we do not have further evidence from deeper observations.

- The metallicity does not show any gradient along the bar, regardless of the SFR gradients and fluctuations described above. However, the metallicity increases significantly with time all along the bar.

Acknowledgements. We warmly thank the anonymous referee, who helped to improve the presentation of this paper. F.F. thanks the IAC for scientific and financial support for the present work, and Giacinto Iannicola and Ivan Ferraro (INAF OAR) for the help in computing the metallicity-age-distance relation. We acknowledge financial support from the Italian National Institute of Astrophysics and the Ministry of University and Research (grants PRIN-INAF 2011 and PRIN-MIUR 2010LY5N2T), from the IAC (grant 310394) and the Spanish Ministries of Science and Innovation and of Economy and Competitiveness (grants AYA2007-3E3507 and AYA2010-16717).

\section{References}

Aparicio, A., \& Gallart, C. 2004, AJ, 128, 1477

Aparicio, A., \& Hidalgo, S. L. 2009, AJ, 138, 567

Cannon, J. M., O’Leary, E. M., Weisz, D. R., et al. 2012, ApJ, 747, 122

Clementini, G., Held, E. V., Baldacci, L., \& Rizzi, L. 2003, ApJ, 588, L85

de Blok, W. J. G., \& Walter, F. 2000, ApJ, 537, L95

de Blok, W. J. G., \& Walter, F. 2003, MNRAS, 341, L39

Feast, M. W., Whitelock, P. A., Menzies, J. W., \& Matsunaga, N. 2012, MNRAS 421,2998

Fusco, F., Buonanno, R., Bono, G., et al. 2012, A\&A, 548, A129

Gallart, C., Aparicio, A., \& Vilchez, J. M. 1996a, AJ, 112, 1928

Gallart, C., Aparicio, A., Bertelli, G., \& Chiosi, C. 1996b, AJ, 112, 1950

Gallart, C., Aparicio, A., Bertelli, G., \& Chiosi, C. 1996c, AJ, 112, 2596

Gieren, W., Pietrzyński, G., Nalewajko, K., et al. 2006, ApJ, 647, 1056

Hidalgo, S. L., Aparicio, A., Martínez-Delgado, D., \& Gallart, C. 2009, ApJ, 705,704

Hidalgo, S. L., Aparicio, A., Skillman, E., et al. 2011, ApJ, 730, 14

Hidalgo, S. L., Monelli, M., Aparicio, A., et al. 2013, ApJ, 778, 103

Kirby, E. N., Cohen, J. G., Guhathakurta, P., et al. 2013, ApJ, 779, 102

Kroupa, P. 2001, MNRAS, 322, 231

Massey, P., Armandroff, T. E., Pyke, R., Patel, K., \& Wilson, C. D. 1995, AJ, 110,2715

Mateo, M. L. 1998, ARA\&A, 36, 435

Pietrinferni, A., Cassisi, S., Salaris, M., \& Castelli, F. 2004, ApJ, 612, 190

Salaris, M., \& Cassisi, S. 1998, MNRAS, 298, 166

Schlafly, E. F., \& Finkbeiner, D. P. 2011, ApJ, 737, 103

Schlegel, D. J., Finkbeiner, D. P., \& Davis, M. 1998, ApJ, 500, 525

Schroyen, J., De Rijcke, S., Koleva, M., Cloet-Osselaer, A., \& Vandenbroucke, B. 2013, MNRAS, 434, 888

Sibbons, L. F., Ryan, S. G., Cioni, M.-R. L., Irwin, M., \& Napiwotzki, R. 2012, A\&A, 540, A135

Sirianni, M., Jee, M. J., Benìtez, N., et al. 2005, PASP, 117, 1049

Stetson, P. B. 1987, PASP, 99, 191

Stetson, P. B. 1994, PASP, 106, 250

Stetson, P. B., \& Harris, W. E. 1988, AJ, 96, 975

van den Bergh, S. 1998, Galaxy Morphology and Classification (Cambridge: Cambridge University Press)

Venn, K. A., Lennon, D. J., Kaufer, A., et al. 2001, ApJ, 547, 765 